\title{
Lattice Boltzmann method for simulation of compressible flows on standard lattices
}

\author{
Nikolaos I. Prasianakis ${ }^{1, *}$ and Iliya V. Karlin ${ }^{1,2, \dagger}$ \\ ${ }^{1}$ Aerothermochemistry and Combustion Systems Laboratory, ETH Zurich, 8092 Zurich, Switzerland \\ ${ }^{2}$ School of Engineering Sciences, University of Southampton, Southampton, SO17 1BJ United Kingdom \\ (Received 11 February 2008; revised manuscript received 15 May 2008; published 21 July 2008)
}

\begin{abstract}
The recently introduced lattice Boltzmann model for thermal flow simulation on a standard lattice [Prasianakis and Karlin, Phys. Rev. E 76, 016702 (2007)] is studied numerically in the case where compressibility effects are essential. It is demonstrated that the speed of sound and shock propagation are described correctly in a wide temperature range, and that it is possible to take into account additional physics such as heat sources and sinks. A remarkable simplicity of the model makes it viable for engineering applications in subsonic flows with large temperature and density variations.
\end{abstract}

DOI: 10.1103/PhysRevE.78.016704

PACS number(s): 47.11.-j, 05.70.Ln

\section{INTRODUCTION}

The lattice Boltzmann (LB) method is a powerful approach to hydrodynamics, with applications ranging from large-Reynolds-number flows to flows at a micrometer scale, porous media, and multiphase flows [1]. The LB method solves a fully discrete kinetic equation for populations $f_{i}(x, t)$, designed to reproduce the Navier-Stokes equations in the hydrodynamic limit. Populations correspond to discrete velocities $c_{i}, i=1, \ldots, N$, which fit into a regular spatial lattice with the nodes $x$. This enables a simple and highly efficient "stream along links and equilibrate at nodes" realization of the LB algorithm.

The success of the LB method is primarily in the incompressible flow simulation. In that case, the LB models on the so-called standard lattices with a relatively small number of velocities $[N=9$ in two dimensions (see Fig. 1) and $N$ $=15,19,27$ in three $]$ are available and most commonly used. In this paper, we shall follow the usual nomenclature and indicate the models as $D M Q N$ where $M=2,3$ is the spatial dimension and $N$ the number of discrete velocities of the model. In the standard LB models for incompressible flow, geometric constraints associated with the lattice are an obstacle to extending the method to compressible flows [2].

Recently, a thermal model was introduced on the standard D2Q9 lattice [3], as an extension of the LB model with energy conservation [4]. The essence of the construction [3] is to introduce counterterms into the kinetic equation in such a way that the constraints due to the lattice are compensated, and the desired compressible Navier-Stokes and energy equations are recovered in the hydrodynamic limit. It was shown that thermal flows with small density variations can be simulated accurately.

The goal of the present paper is to access, through a carefully chosen set of benchmark problems, the efficiency of the LB method [3] for compressible flows. We are especially interested in subsonic flows with large density and temperature variations, such as those pertinent to combustion. The

\footnotetext{
*Present address: Paul Scherrer Institute, Combustion Research, CH-5232 Villigen PSI, Switzerland. Nikolaos.Prasianakis@psi.ch

†Corresponding author. karlin@lav.mavt.ethz.ch
}

outline of the paper is as follows: In Sec. II and the Appendix, for the sake of completeness, we review the LB model of Ref. [3]. In Sec. III, the speed of sound is measured in order to verify the lack of dependence of the model on a reference temperature. In Sec. IV, the results of shock tube simulations are presented. In Sec. V, a model compressible flow with heat sources and sinks is considered in order to examine compressibility effects at large temperature variations. Finally, results are discussed in Sec. VI.

\section{THERMAL LATTICE BOLTZMANN ALGORITHM}

A. Entropic lattice Boltzmann model with energy conservation

We begin by recalling the construction of the local equilibrium in the thermal lattice Boltzmann model [3-5] on a planar square lattice with nine velocities $c_{i \alpha}, i=0, \ldots, 8$ (the $D 2 Q 9$ lattice; see Fig. 1):

$$
\begin{aligned}
& c_{x}=\{0,1,0,-1,0,1,-1,-1,1\}, \\
& c_{y}=\{0,0,1,0,-1,1,1,-1,-1\} .
\end{aligned}
$$

The equilibrium populations are obtained from minimization of the entropy function $H$ under specific constraints. For the $D 2 Q 9$ model, the entropy function has the form [6]

$$
H=\sum_{i=0}^{8} f_{i} \ln \left(\frac{f_{i}}{W_{i}}\right),
$$

where the weights $W_{i}$ are

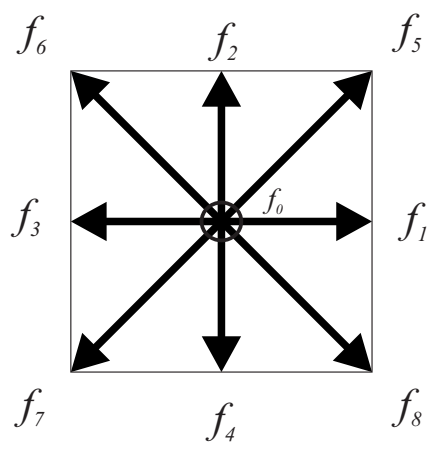

FIG. 1 . The $D 2 Q 9$ velocity set. 


$$
W=\frac{1}{36}\{16,4,4,4,4,1,1,1,1\} .
$$

The constraints of the minimization problem are the local conservation laws of mass, momentum, and energy,

$$
\begin{gathered}
\sum_{i=0}^{8} f_{i}^{\mathrm{eq}}=\sum_{i=0}^{8} f_{i}=\rho, \\
\sum_{i=0}^{8} c_{i \alpha} f_{i}^{\mathrm{eq}}=\sum_{i=0}^{8} c_{i \alpha} f_{i}=j_{\alpha}, \\
\sum_{i=0}^{8} c_{i}^{2} f_{i}^{\mathrm{eq}}=\sum_{i=0}^{8} c_{i}^{2} f_{i}=2 \rho T+\frac{j^{2}}{\rho},
\end{gathered}
$$

where $\rho, j_{\alpha}=\rho u_{\alpha}$, and $T$ are the density, momentum, and temperature fields, respectively. This results in the local equilibrium with energy conservation $[4,5]$. Deviation of the equilibrium pressure tensor $P_{\alpha \beta}^{\mathrm{eq}}=\sum_{i=0}^{8} c_{i \alpha} c_{i \beta} \int_{i}^{\mathrm{eq}}$ from the Maxwell-Boltzmann form

$$
P_{\alpha \beta}^{\mathrm{MB}}=\rho T \delta_{\alpha \beta}+\frac{j_{\alpha} j_{\beta}}{\rho}
$$

is of the order $j^{2} \Delta T$, where $\Delta T$ is equal to $\Delta T=T-T_{0}$ and $T_{0}=1 / 3$ is the reference temperature in lattice units. In order to remove the dependence of the equilibrium pressure tensor from the reference temperature, the minimization problem is modified. For that, guided equilibrium populations are introduced.

\section{B. Guided equilibrium}

An additional condition, which generates the so-called guided equilibrium populations [3,7], stipulates that the equilibrium pressure tensor $P_{\alpha \beta}^{\mathrm{eq}}$ is in the Maxwell-Boltzmann form:

$$
\sum_{i=0}^{8} c_{i \alpha} c_{i \beta} f_{i}^{\mathrm{eq}}=\rho T \delta_{\alpha \beta}+\frac{j_{\alpha} j_{\beta}}{\rho}
$$

Details of the derivation of the equilibrium populations as a minimizer of the entropy function (2) under constraints (4) and (6) are provided in Ref. [3]. The guided equilibrium populations $f_{i}^{\mathrm{eq}}(\rho, \mathbf{u}, T)$, expanded up to the fourth order in velocity, have a simple form:

$$
f_{i}^{\mathrm{eq}}=\rho \prod_{\alpha=x, y} \frac{\left(1-2 c_{i \alpha}^{2}\right)}{2^{c_{i \alpha}^{2}}}\left[\left(c_{i \alpha}^{2}-1\right)+c_{i \alpha} u_{\alpha}+u_{\alpha}^{2}+T\right] .
$$

Note that at $u=0$ equilibrium populations are positive if the temperature $T$ is restricted to the interval $T \in[0,1]$ (in lattice units).

\section{Introduction of counterterms}

Third- and fourth-order moments of the equilibrium populations (7) are not exact compared to the corresponding moments of the local Maxwell distribution function whenever the temperature deviates from the reference value $T_{0}=1 / 3$ $[3,4]$. In order to alleviate this limitation, the method introduced in [3] is used. First, correction terms $\Psi_{i}$ and $\Phi_{i}$ are introduced into the Boltzmann equation with the BhatnagarGross-Krook collision operator,

$$
\partial_{t} f_{i}+c_{i \alpha} \partial_{\alpha} f_{i}=-\frac{1}{\tau}\left(f_{i}-f_{i}^{\mathrm{eq}}\right)+\Psi_{i}+\Phi_{i} .
$$

The latter terms are defined using vectors $\Psi_{x}, \Psi_{y}$, and $\Phi$ :

$$
\begin{gathered}
\Psi_{x}=\frac{1}{4}[0,4,0,-4,0,-1,1,1,-1], \\
\Psi_{y}=\frac{1}{4}[0,0,4,0,-4,-1,-1,1,1], \\
\Phi=\frac{1}{8}[-12,4,4,4,4,-1,-1,-1,-1] .
\end{gathered}
$$

The correction terms are tailored in such a way as to compensate for the deviations appearing in the momentum and energy equations,

$$
\begin{gathered}
\Psi_{i}=\Psi_{i \alpha} \partial_{\kappa} P_{\alpha \kappa}^{\prime \prime}, \\
\Phi_{i}=\Phi_{i} \partial_{\alpha}\left(q_{\alpha}^{\prime}+q_{\alpha}^{\prime \prime}\right),
\end{gathered}
$$

where $\partial_{\kappa} P_{\alpha \kappa}^{\prime \prime}$ is the deviation in the momentum and $\partial_{\alpha}\left(q_{\alpha}^{\prime}\right.$ $\left.+q_{\alpha}^{\prime \prime}\right)$ in the energy equations. Deviation terms are identified through the Chapman-Enskog analysis as presented in Ref. [3]. Here only the final result is reproduced:

$$
\begin{aligned}
\partial_{\gamma} P_{x \gamma}^{\prime \prime}= & -\frac{\tau}{2} \partial_{x}\left[\partial_{x}\left(j_{x}(1-3 T)-\frac{j_{x}^{3}}{\rho^{2}}\right)-\partial_{y}\left(j_{y}(1-3 T)-\frac{j_{y}^{3}}{\rho^{2}}\right)\right] \\
& +\rho g_{x}, \\
\partial_{\gamma} P_{y \gamma}^{\prime \prime}= & -\frac{\tau}{2} \partial_{y}\left[\partial_{y}\left(j_{y}(1-3 T)-\frac{j_{y}^{3}}{\rho^{2}}\right)-\partial_{x}\left(j_{x}(1-3 T)-\frac{j_{x}^{3}}{\rho^{2}}\right)\right] \\
& +\rho g_{y},
\end{aligned}
$$

$$
\begin{gathered}
q_{\alpha}^{\prime}=j_{\alpha}(1-3 T)-\frac{j_{\alpha}^{3}}{\rho^{2}}, \\
q_{\alpha}^{\prime \prime}=\frac{(4-\operatorname{Pr})}{\operatorname{Pr}} \tau \rho T \partial_{\alpha} T-\tau\left[\frac{3 j_{\alpha} j_{\alpha}}{\rho^{2}} \partial_{\alpha}(\rho T)+3 j_{\alpha} T \partial_{\beta}\left(\frac{j_{\beta}}{\rho}\right)\right. \\
\left.-\frac{3 j_{\alpha} j_{\beta}}{2 \rho} \partial_{\beta} T\right]-\tau\left\{(1-3 T)\left[\partial_{\beta}\left(\frac{j_{\alpha} j_{\beta}}{\rho}\right)-\frac{j_{\alpha}}{2 \rho} \partial_{\beta} j_{\beta}\right]\right\} \\
-\tau\left[2 \frac{j_{\alpha}^{3}}{\rho^{3}} \partial_{\beta} j_{\beta}+\frac{3 j_{\alpha} j_{\alpha}}{\rho^{2}} \partial_{\beta}\left(\frac{j_{\alpha} j_{\beta}}{\rho}\right)+\frac{j_{\alpha}}{2 \rho} \partial_{\beta}\left(\frac{j_{\beta}^{3}}{\rho^{2}}\right)\right]+\tau \partial_{\beta} e_{\alpha \beta}^{\prime \prime}, \\
e_{x x}^{\prime \prime}=\frac{j_{x} j_{x}}{\rho}+\frac{j_{x}^{4}}{\rho^{3}}, \quad e_{y y}^{\prime \prime}=\frac{j_{y} j_{y}}{\rho}+\frac{j_{y}^{4}}{\rho^{3}}, \quad e_{x y}^{\prime \prime}=\frac{j_{x} j_{y} j^{2}}{\rho^{3}} . \quad \text { (12) }
\end{gathered}
$$

Here, $g_{x}$ and $g_{y}$ represent acceleration due to external forces such as gravity, and Pr is the Prandtl number. 


\section{Hydrodynamic limit}

After the introduction of the counterterms into the kinetic equation (8) as explained above, the latter recovers the compressible Navier-Stokes equations in the hydrodynamic limit:

$$
\begin{gathered}
\partial_{t} \rho=-\partial_{\gamma}\left(\rho u_{\gamma}\right) \\
\partial_{t} u_{\alpha}=-u_{\gamma} \partial_{\gamma} u_{\alpha}-\frac{1}{\rho} \partial_{\alpha}(\rho T)+\frac{1}{\rho} \partial_{\gamma}\left[\tau \rho T \left(\partial_{\alpha} u_{\gamma}+\partial_{\gamma} u_{\alpha}\right.\right. \\
\left.\left.-\partial_{\kappa} u_{\kappa} \delta_{\alpha \gamma}\right)\right]+\rho g_{\alpha}, \\
\partial_{t} T=-u_{\alpha} \partial_{\alpha} T-T \partial_{\alpha} u_{\alpha}+\frac{1}{\rho}\left[\partial_{\alpha}\left(\frac{2}{\operatorname{Pr}} \tau \rho T \partial_{\alpha} T\right)\right. \\
\left.+\left(\partial_{\gamma} u_{\alpha}\right) \tau \rho T\left(\partial_{\alpha} u_{\gamma}+\partial_{\gamma} u_{\alpha}-\partial_{\kappa} u_{\kappa} \delta_{\alpha \gamma}\right)\right]
\end{gathered}
$$

The corresponding fluid obeys the ideal gas equation of state, $p=\rho T$, with the adiabatic exponent $\gamma=c_{p} / c_{v}=2$. The viscosity coefficient $\mu$ and thermal conductivity $\kappa$ are identified as

$$
\mu=\tau \rho T, \quad \kappa=\frac{2}{\operatorname{Pr}} \tau \rho T
$$

The Prandtl number $\operatorname{Pr}=c_{p} \mu / \kappa$, is a parameter of the model.

\section{E. The lattice Boltzmann scheme}

The discretization in time and space of the kinetic equation (8) results in an implicit scheme, not very different from the standard lattice Boltzmann formulation. The lattice Bhatnagar-Gross-Krook equation with a transformation similar to the one introduced in [8] reads

$$
\begin{gathered}
g_{i}=f_{i}+\frac{\delta t}{2 \tau}\left(f_{i}-f_{i}^{\mathrm{eq}}\right)-\frac{\delta t}{2}\left(\Psi_{i}+\Phi_{i}\right), \\
g_{t+\delta t}=g_{t}+\frac{2 \delta t}{\delta t+2 \tau}\left[f_{t}^{\mathrm{eq}}-g_{t}\right]+\frac{2 \tau \delta t}{\delta t+2 \tau}\left(\Psi_{t}+\Phi_{t}\right),
\end{gathered}
$$

where the time step $\delta t$ in the standard lattice Boltzmann formulation is set as $\delta t=1$. Computation is carried out on the level of the transformed populations $g$. The equilibrium populations $f^{\mathrm{eq}}$, needed in Eq. (16), are computed with the help of the moments of Eq. (15), which relate the locally conserved moments of the populations $f$ with the moments of the $g$ populations. It is straightforward to show that the density $\rho(f)$, the components of the momentum $j_{x}(f)$ and $j_{y}(f)$, and the temperature $T(f)$ can be evaluated as follows:

$$
\begin{gathered}
\rho(f)=\rho(g), \\
j_{x}(f)=j_{x}(g)+\frac{\delta t}{2}\left[\partial_{\gamma} P_{x \gamma}^{\prime \prime}(f)\right], \\
j_{y}(f)=j_{y}(g)+\frac{\delta t}{2}\left[\partial_{\gamma} P_{y \gamma}^{\prime \prime}(f)\right],
\end{gathered}
$$

$$
T(f)=\frac{1}{2 \rho}\left(\sum_{i=0}^{8} c_{i}^{2} g_{i}-\frac{j^{2}(f)}{\rho}+\frac{\delta t}{2} \partial_{\alpha}\left[q_{\alpha}^{\prime}(f)+q_{\alpha}^{\prime \prime}(f)\right]\right) .
$$

The lattice Boltzmann scheme of Eq. (16) is semiexplicit due to the presence of the correction terms $\Psi_{i}$ and $\Phi_{i}$ (and not fully explicit, as in the standard case without any corrections). The following algorithm is implemented for the collision step.

Step 1. Calculate $\rho, j_{\alpha}, T$ using (17)-(20), $\left(\partial_{\gamma} P_{\alpha \gamma}^{\prime \prime}\right)^{t-1}$, $\left[\partial_{\alpha}\left(q_{\alpha}^{\prime}+q_{\alpha}^{\prime \prime}\right)\right]^{t-1}$ and $g_{t}$ values.

Step 2. Calculate $\left(\partial_{\gamma} P_{\alpha \gamma}^{\prime \prime}\right)^{t},\left[\partial_{\alpha}\left(q_{\alpha}^{\prime}+q_{\alpha}^{\prime \prime}\right)\right]^{t}$ using values of $\rho, j_{\alpha}, T$ from step 1 .

Step 3. Calculate again $\rho, j_{\alpha}, T$ using (17)-(20), $g_{t}$, and the values calculated in step 2 .

Step 4. Use $\rho, j_{\alpha}, T$ from step 3 for the calculation of the equilibrium values (7).

Step 5. Use $\left(\partial_{\gamma} P_{\alpha \gamma}^{\prime \prime}\right)^{t},\left(\partial_{\alpha}\left(q_{\alpha}^{\prime}+q_{\alpha}^{\prime \prime}\right)\right)^{t}$ from step 2 in the discrete equation (16) along with the equilibrium values calculated in step 4.

The terms $\partial_{\gamma} P_{\alpha \gamma}^{\prime \prime}$ and $\partial_{\alpha}\left(q_{\alpha}^{\prime}+q_{\alpha}^{\prime \prime}\right)$ can be evaluated using a second-order accurate central finite-difference scheme (see the Appendix).

\section{REFERENCE TEMPERATURE INDEPENDENCE}

The notion of the reference temperature $T_{0}$ is pertinent to most of the existing thermal lattice Boltzmann models. If the temperature is set to the reference value, deviations of the higher-order moments of the corresponding equilibrium from the moments of the Maxwellian are minimized. The value of the reference temperature $T_{0}$ is different for different LB models. When these models are operated in a small window around the reference temperature, deviation terms in the higher-order moments of the equilibrium populations are minimized. Thus, the simulation of weakly compressible flows is possible. For example, the accuracy of the $D 2 Q 9$ model with energy conservation [4,5] as well as of the guided equilibrium model described by (7) [that is, without introducing the correction terms in (8)] depends on the temperature deviation from the reference temperature $T_{0}=1 / 3$. For larger velocity sets, deviation terms are still present in the equilibrium moments and the situation is not qualitatively different. For example, in the 17-velocity $D 2 Q 17$ model of Refs. [9-11], deviation terms are of the order $O\left(\Theta u^{2}\right)$, where $\Theta=\left(T / T_{0}\right)-1$, and $T_{0}$ is the reference temperature of the $D 2 Q 17$ model. Not surprisingly, as reported in Ref. [10], the error increases as the temperature deviates from the reference temperature and the model becomes unstable even for small temperature gradients. An improved LB model with 37 velocities (the D2Q37 lattice, Ref. [9]) still contains deviations, among others, of the order $O\left(\Theta^{2} u\right)$. Thus, as soon as large temperature variations are encountered in a simulation, the deviation terms may become large, resulting in an incorrect behavior or even instabilities. For achieving a better accuracy, lattices with a large number of velocities were suggested (e.g., the D2Q53 and D2Q81 models, Refs. [9-11]). It should be recalled, however, that com- 


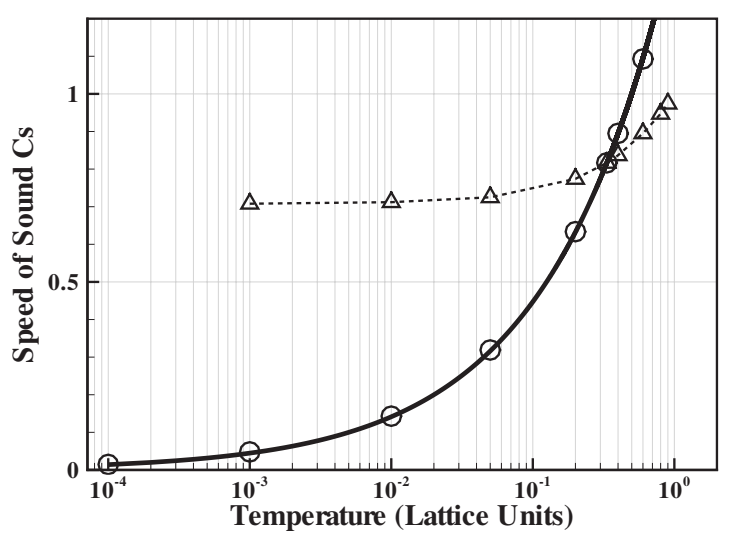

FIG. 2. Speed of sound for different temperatures (logarithmic scale; temperature is given in lattice units). Circles: Simulation results of the thermal model of Sec. II. Triangles: Simulation results of the LB model with energy conservation but without correction terms [4]. Solid line: Theoretical prediction $c_{\mathrm{S}}=\sqrt{2 T}$.

putational complexity increases as soon as more velocities are introduced, e.g., for boundary condition implementation.

In order to work with standard lattices, the introduction of correction terms [3] which remove the existing deviation is absolutely necessary. After the introduction of these terms, the validity of the model is not dependent on the reference temperature $T_{0}=1 / 3$ any longer. In order to demonstrate that the LB model of Sec. II allows indeed for a range of temperatures, the speed of sound for different temperatures is measured through a simulation. The speed of sound $c_{\mathrm{s}}$ is defined as the speed of propagation of a small pressure perturbation. In order to create a sound wave, a quasi-onedimensional slab split into two regions is considered. The temperature is kept uniform, but different pressure and density in each half of the simulation domain is imposed. The pressure difference between the left half of the domain $P_{L}$ and the right half of the domain $P_{R}$ is set to $\left(P_{L}-P_{R}\right) / P_{R}$ $=10^{-4}$. A grid size of 3000 nodes in the $x$ direction and five nodes in the $y$ direction was used. Periodic boundary conditions in the $y$ direction were applied. The Prandtl number was set to $\operatorname{Pr}=1$.

As soon as the simulation starts, a discontinuity propagates in the domain, and by monitoring its location the speed of sound is measured. The theoretical value of the speed of sound for the model of Sec. II is $c_{\mathrm{s}}=\sqrt{\gamma T}$, where the specific heat ratio is $\gamma=2$. A plot of the speed of sound for different temperatures $T \in] 0,1]$ (in lattice units) is presented in Fig. 2. Excellent agreement between the simulation results and the theoretical prediction is observed in a range of temperatures above and below the reference temperature $T_{0}=1 / 3$. In particular, the agreement stays excellent even if the temperature is several orders less than $T_{0}$. For the sake of comparison, the result for the LB model with energy conservation but without correction terms [4] is also presented in Fig. 2. As expected, the speed of sound for the latter LB model crosses the theoretical curve only at the reference temperature $T_{0}=1 / 3$.

Thus, we verified numerically that the present thermal model is indeed reference temperature independent. The leading order counterterm that allows the accurate descrip- tion of the speed of sound is the term $\partial_{\alpha}\left(q_{\alpha}^{\prime}\right)$, which guarantees the accurate advection of the heat flux $q_{\alpha}$. The lattice temperature can now be lowered, resulting in a lower value of the speed of sound compared to the population's advection velocity which is equal to 1 . Since the physics is described correctly in a wide temperature range, the upper bound for the temperature $T$ can be shifted closer to zero. This makes possible the simulation of flows where temperature gradients of the order $O(10)$ or higher are present, while at the same time the temperature stays inside the interval $[0,1]$, as will be illustrated in Sec. V.

\section{SHOCK TUBE SIMULATION}

Compressible flow simulation within the present $D 2 Q 9$ thermal model was studied further with the shock tube problem. The setup is similar to that of Sec. III. The onedimensional slab consists of two separated domains which contain the same gas at different values of density, pressure, and temperature. Domains are separated by a barrier that is located in the middle of the domain. As soon as the barrier is removed, two waves start propagating away from the discontinuity: a shock wave traveling into the lower-pressure region, and a rarefaction wave moving into the higher-pressure region. The initial conditions for the simulation are $T_{L}=0.4$, $P_{L}=0.2$, and $\rho_{L}=0.5$ for the left region and $T_{R}=0.05, P_{R}$ $=0.1$, and $\rho_{R}=2$ for the right region. Thus, the pressure ratio between the domains is set to 2 , and the temperature ratio is 8 . A $3000 \times 5$ grid was used; periodic boundary condition in the $y$-direction were applied. The Prandtl number was set to $\operatorname{Pr}=1$ and the relaxation parameter $\tau=0.2$.

Simulation results for the thermal model of Sec. II are compared to the analytical solution in Fig. 3. The four plots in Fig. 3 show the density, the temperature, the pressure, and the local Mach number of the flow, respectively, at time $t$ $=900$ in lattice units. The local Mach number is defined as the ratio between the velocity and the speed of sound at a given point, $\operatorname{Ma}(x, t)=u(x, t) / c_{\mathrm{S}}(x, t)$. In the present simulation, the local Mach number reaches the value $\mathrm{Ma}_{\max }$ $=0.235$ behind the shock wave. Excellent agreement of the simulation results with the analytical solution can be observed for all four quantities. It should be stressed that no stabilization procedure was used in the current simulations.

\section{COMPRESSIBLE FLOW WITH DISTRIBUTED HEAT SOURCES AND SINKS}

In this section, we validate the present model by constructing a flow inspired by Ref. [13]. We consider a stationary unidirectional solution to the Navier-Stokes and energy equations supplemented with additional terms in the momentum and energy equations. In the energy equation, a term $\dot{w}_{0}(x)$ is introduced, while the term $\dot{H}_{0}(x)$ appears in the momentum equation. Considering the flow at the steady state and periodic boundary conditions in the $y$ direction, the system is

$$
u_{x} \partial_{x} T_{x}=-T_{x} \partial_{x} u_{x}+\frac{\kappa}{\rho} \partial_{x} \partial_{x} T+\frac{1}{2 \rho} \dot{w}_{0},
$$



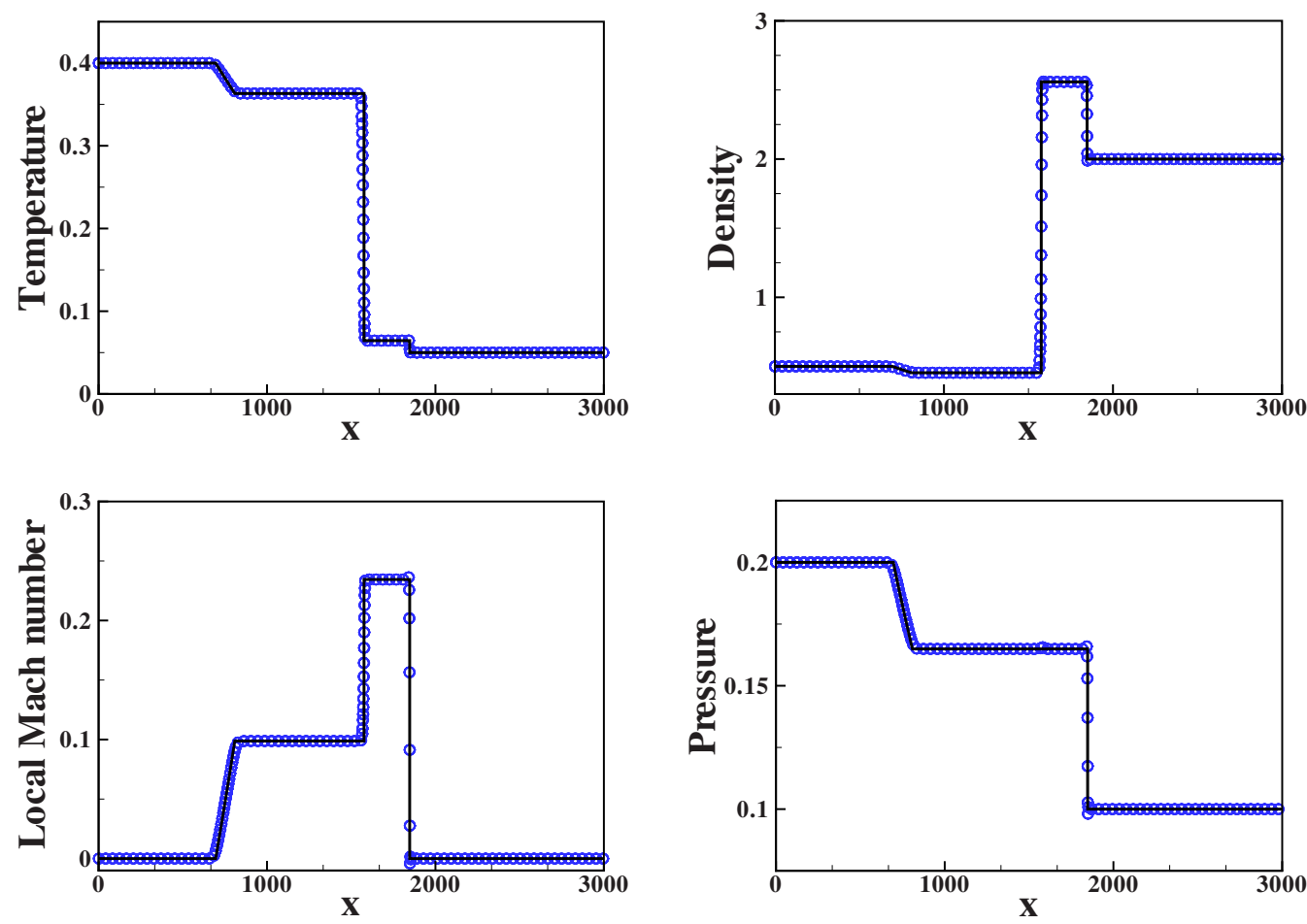

FIG. 3. (Color online) Shock tube simulation after $t=900$ time steps (lattice units). Comparison with the analytical solution for density, temperature, pressure, and local Mach number of the flow. Line, analytical solution, Ref. [12]; symbols, thermal $D 2 Q 9$ LB model of Sec. II.

$$
\begin{gathered}
u_{x} \partial_{x} u_{x}=-\frac{1}{\rho} \partial_{x}(\rho T)+\frac{\mu}{\rho} \partial_{x} \partial_{x} u_{x}+\frac{1}{\rho} \dot{H}_{0}, \\
\partial_{x}\left(\rho u_{x}\right)=0 .
\end{gathered}
$$

The system (21) is considered on the interval $x \in[-1,1]$. The viscosity $\mu$ and the heat conductivity $\kappa$ are assumed constant.

At the inflow $(x=-1)$ and at the outflow $(x=1)$ the following boundary conditions are imposed for the temperature and the velocity:

$$
\begin{aligned}
& T_{x}( \pm 1)=\frac{1}{2} T_{c}\left[1.2+\tanh \left(\frac{ \pm 1}{\delta}\right)\right], \\
& u_{x}( \pm 1)=\frac{1}{2} U_{c}\left[1.2+\tanh \left(\frac{ \pm 1}{\delta}\right)\right] .
\end{aligned}
$$

Here $T_{c}$ and $U_{c}$ are the characteristic temperature and velocity of the flow.

Following Ref. [13], the solution of the system (21) mimics a premixed flame located at $x=0$. The reactants approach the flame from the inflow $(x=-1)$, while the products exit the domain at the outflow $(x=1)$. The parameter $\delta$ can be considered as the flame thickness, and the velocity and temperature profiles depend on that parameter. The term $\dot{w}_{0}$ in the energy equation is a sum of two contributions, $\dot{w}_{0}=\dot{w}_{1}+\dot{w}_{2}$, where

$$
\dot{w}_{1}=\rho \frac{T_{c} U_{c}}{\delta} \operatorname{sech}^{2}\left(\frac{x}{\delta}\right)\left[1.2+\tanh \left(\frac{x}{\delta}\right)\right],
$$

$$
\dot{w}_{2}=2 \frac{\kappa T_{c}}{\delta^{2}} \tanh \left(\frac{x}{\delta}\right) \operatorname{sech}^{2}\left(\frac{x}{\delta}\right) .
$$

Here, $\dot{w}_{1}$ represents distributed heat sources with a maximum located at the center of the domain, while $\dot{w}_{2}$ represents heat sinks at the leftmost part of the domain and heat sources at the rightmost part. As heating due to viscous dissipation is negligible, it is not taken into account in the energy equation. Furthermore, in order to obtain the steady state solution of (25), it is required that the thermodynamic pressure $p$ remains constant in the domain. This is achieved upon introducing the term $\dot{H}_{0}$ in the momentum equation:

$$
\dot{H}_{0}=\frac{U_{c} \operatorname{sech}^{2}(x / \delta)\left[1.2 U_{c} \rho \delta+\left(4 \mu+U_{c} \rho \delta\right) \tanh (x / \delta)\right]}{4 \rho \delta^{2}} .
$$

Finally, the analytical solution to the system (21) is the following:

$$
\begin{aligned}
& u_{x}=\frac{1}{2} U_{c}\left[1.2+\tanh \left(\frac{x}{\delta}\right)\right], \\
& T_{x}=\frac{1}{2} T_{c}\left[1.2+\tanh \left(\frac{x}{\delta}\right)\right] .
\end{aligned}
$$

In order to simulate the aforementioned flow with the present thermal LB model, we need to take into account the above heat sources and sinks, as well as the additional terms in the momentum equation. This is done conveniently by altering the correction terms (10) in the kinetic equation: 


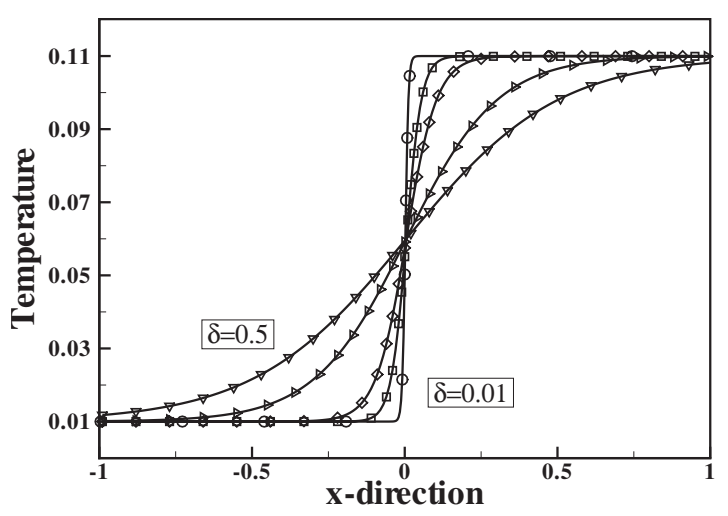

FIG. 4. Temperature plots for $\delta=(0.01,0.05,0.1,0.3,0.5), T_{c}$ $=0.1$, and $U_{c}=0.01$ in lattice units. Solid line represents the analytical solution, while symbols are the thermal lattice Boltzmann simulation.

$$
\begin{gathered}
\Psi_{i}=\Psi_{i \alpha}\left(\partial_{\kappa} P_{\alpha \kappa}^{\prime \prime}+\rho \dot{H}_{0} \delta_{\alpha x}\right), \\
\Phi_{i}=\Phi_{i}\left(\partial_{\alpha} q_{\alpha}^{\prime}+\partial_{\alpha} q_{\alpha}^{\prime \prime}+2 \rho \dot{w}_{0}\right) .
\end{gathered}
$$

In Figs. 4 and 5, simulation results are compared with the steady state analytical solution. A $200 \times 5$ grid was used. In the $x$ direction, for the first and last nodes, the conditions of Eq. (22) were imposed by assigning equilibrium values for the populations. Periodic conditions were implemented in the $y$ direction. The characteristic temperature for the simulation presented in Figs. 4 and 5 is $T_{c}=0.1$, the characteristic velocity is $U_{c}=0.01$, the viscosity coefficient is $\mu=0.01$, and the Prandtl number is set to $\operatorname{Pr}=1$. The parameter $\delta$ is varied, $\delta=(0.01,0.05,0.1,0.3,0.5)$. The temperature ratio between the inlet and the outlet depends slightly on the value of $\delta$ and takes values $T_{x}(+1) / T_{x}(-1)=(11,11,11,10.9,9.2)$, respectively; that is, the temperature ratio is of the order of ten in the present simulation. For the initial condition, we take the equilibrium with the linear temperature and velocity profile between the values at the inlet $\left[T_{x}(-1), U_{x}(-1)\right]$ and the values at the outlet $\left[T_{x}(+1), U_{x}(+1)\right]$. As it is clearly seen in Figs. 4 and 5 , the results of simulations are in excellent agreement with the analytical solution (25).

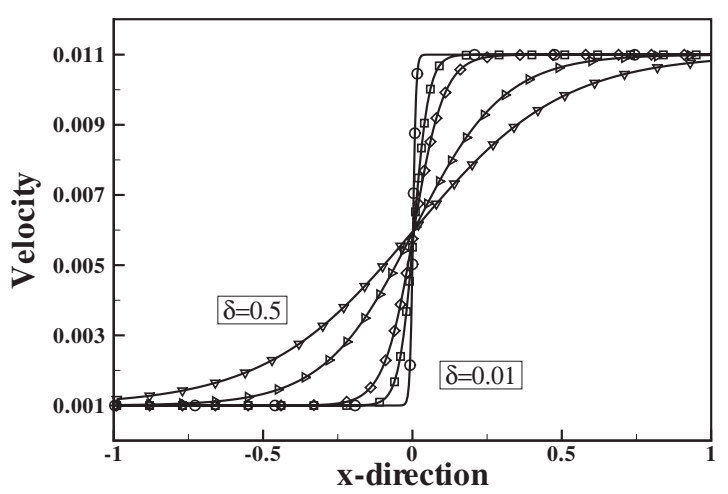

FIG. 5. Velocity plots for $\delta=(0.01,0.05,0.1,0.3,0.5), T_{c}=0.1$, and $U_{c}=0.01$ in lattice units. Solid line represents the analytical solution, while symbols are the thermal lattice Boltzmann simulation.
The study of the system presented in this section reveals some interesting aspects of the model. Depending on the characteristic parameters of the flow $\left(U_{c}, T_{c}, \delta\right.$, and Pr), the term $\dot{w}_{1}$ can be the leading order term, $\dot{w}_{1} \gg \dot{w}_{2}(\operatorname{Pr} \gg 1)$, thus validating the correct advection of the heat flux in the energy equation. On the other hand, if the term $\dot{w}_{2}$ is dominant, $\dot{w}_{1}$ $\ll \dot{w}_{2}(\operatorname{Pr} \ll 1)$, the correctness of the heat diffusion (the Fourier law) is validated. In the simulation, the Prandtl number was varied in the range $10^{-4}-10^{4}$ without affecting either the stability or the accuracy of the results.

\section{CONCLUSION}

In this paper, the thermal $D 2 Q 9$ model introduced in [3] was validated in flow situations which are characterized by large temperature and density variations. Deviation terms present in the higher-order moments of the equilibrium populations, emerging from the low symmetry of the $D 2 Q 9$ lattice, are removed. This provides increased accuracy and stability of the model, while additional terms such as heat sources and sinks can be taken into account easily. In two dimensions, the nine populations are found to be adequate for a successful simulation of subsonic compressible flows, thus keeping the complexity of the algorithm at a low level. Characteristic properties, such as the speed of sound, can be described in a broad temperature range covering almost four orders of magnitude. Shock tube simulations provide an insight about the stability of the model, given that even eight times temperature difference steps can be simulated accurately. The compressible benchmark flow with heat sources and sinks verifies that both the heat flux advection and that the heat diffusion in the energy equation are represented correctly by the lattice Boltzmann model. In all cases the ideal gas equation of state is verified. Taking into account these results, the present model can be considered as a successful implementation of the lattice Boltzmann algorithm to simulate subsonic fully compressible flows characterized by large temperature and density variations at the mere computational cost of the D2Q9 lattice. The efficiency, stability, and accuracy of the current model make it promising for the study of standard engineering applications. Application of the model for combustion problems as well as for thermal mixing problems is currently under consideration.

\section{ACKNOWLEDGMENTS}

The authors thank K. B. Boulouchos, C. Frouzakis, and A. Tomboulides for suggestions and discussions. We acknowledge support by ETH Project No. 0-20235-05 (N.I.P.) and CCEM-CH (I.V.K.).

\section{APPENDIX: DISCRETIZATION OF THE CORRECTION TERMS}

In this Appendix, details pertinent to the implementation of the scheme are presented. The correction terms Eq. (10) are discretized. For that, the spatial derivatives of Eqs. (11) and (12) are evaluated using a second-order-accurate central finite-difference scheme. For example, the discretization of 
the term $\partial_{\alpha} q_{\alpha}^{\prime}=\partial_{x} q_{x}^{\prime}+\partial_{y} q_{y}^{\prime}$ for the $(i, j)$ node, where $i$ and $j$ correspond to the $x$ and $y$ directions, respectively, is done with the help of the following formulas:

$\partial_{x} q_{x}^{\prime(i, j)}=\frac{\left[j_{x}(1-3 T)-j_{x}^{3} / \rho^{2}\right]^{(i+1, j)}-\left[j_{x}(1-3 T)-j_{x}^{3} / \rho^{2}\right]^{(i-1, j)}}{2}$,

$\partial_{y} q_{y}^{\prime(i, j)}=\frac{\left[j_{y}(1-3 T)-j_{y}^{3} / \rho^{2}\right]^{(i, j+1)}-\left[j_{y}(1-3 T)-j_{y}^{3} / \rho^{2}\right]^{(i, j-1)}}{2}$.

Similar expressions are easily obtained also for the momentum equation correction. To achieve better accuracy in the evaluation of flux terms in the energy equation, a finitedifference scheme with a larger stencil can be used even though this does not change the overall second-order accuracy of the scheme. For example, for the correction terms $\partial_{x} q_{x}^{\prime}$ and $\partial_{y} q_{y}^{\prime}$, the following relations were used:

$\left(\partial_{x} q_{x}^{\prime}\right)^{(i, j)}=\frac{-\left(q_{x}^{\prime}\right)^{(i+2, j)}+8\left(q_{x}^{\prime}\right)^{(i+1, j)}-8\left(q_{x}^{\prime}\right)^{(i-1, j)}+\left(q_{x}^{\prime}\right)^{(i-2, j)}}{12}$,

[1] S. Succi, The Lattice Boltzmann Equation for Fluid Dynamics and Beyond (Oxford University Press, Oxford, 2001).

[2] S. Succi, I. V. Karlin, and H. Chen, Rev. Mod. Phys. 74, 1203 (2002).

[3] N. I. Prasianakis and I. V. Karlin, Phys. Rev. E 76, 016702 (2007).

[4] S. Ansumali and I. V. Karlin, Phys. Rev. Lett. 95, 260605 (2005).

[5] N. I. Prasianakis, S. S. Chikatamarla, I. V. Karlin, S. Ansumali, and K. B. Boulouchos, Math. Comput. Simul. 72, 179 (2006).

[6] I. V. Karlin, A. Ferrante, and H. C. Öttinger, Europhys. Lett. 47, 182 (1999).

[7] I. V. Karlin and S. Succi, Phys. Rev. E 58, R4053 (1998). $\left(\partial_{y} q_{y}^{\prime}\right)^{(i, j)}=\frac{-\left(q_{y}^{\prime}\right)^{(i, j+2)}+8\left(q_{y}^{\prime}\right)^{(i, j+1)}-8\left(q_{y}^{\prime}\right)^{(i, j-1)}+\left(q_{y}^{\prime}\right)^{(i, j-2)}}{12}$.

Finally, the solid wall boundaries are treated as follows. For a two-dimensional rectangular grid, with $n i$ nodes in the $x$ direction and $n j$ nodes in the $y$ direction, two wall boundaries parallel to the $x$ direction are assumed. The lower wall is described by the nodes $(i=1, \ldots, n i, j=1)$ and the upper wall by the nodes $i=1, \ldots, n i, j=n j$. The diffusive wall boundary condition of Ref. [14] can be used. On the boundaries, the derivatives are calculated using an upwind secondorder finite-difference scheme:

$$
\partial_{y} q_{y}^{\prime(i, n j)}=\frac{3\left(q_{y}^{\prime}\right)^{(i, n j)}-4\left(q_{y}^{\prime}\right)^{(i, n j-1)}+\left(q_{y}^{\prime}\right)^{(i, n j-2)}}{2},
$$

$$
\partial_{y} q_{y}^{\prime(i, 1)}=\frac{-\left(q_{y}^{\prime}\right)^{(i, 3)}+4\left(q_{y}^{\prime}\right)^{(i, 2)}-3\left(q_{y}^{\prime}\right)^{(i, 1)}}{2} .
$$

[8] X. He, S. Chen, and G. D. Doolen, J. Comput. Phys. 146, 282 (1998).

[9] P. C. Philippi, L. A. Hegele, L. O. E. dos Santos, and R. Surmas, Phys. Rev. E 73, 056702 (2006).

[10] D. N. Siebert, L. A. Hegele, R. Surmas, L. O. E. Dos Santos, and P. C. Philippi, Int. J. Mod. Phys. C 18, 546 (2007).

[11] P. C. Philippi, L. A. Hegele, R. Surmas, D. N. Siebert, and L. O. E. Dos Santos, Int. J. Mod. Phys. C 18, 556 (2007).

[12] J. D. Anderson, Modern Compressible Flow, 3rd ed. (McGraw-Hill, New York, 2004).

[13] A. G. Tomboulides and S. A. Orszag, J. Comput. Phys. 146, 691 (1998).

[14] S. Ansumali and I. V. Karlin, Phys. Rev. E 66, 026311 (2002). 